\title{
Understanding the three and four-leg inverter Space Vector
}

\author{
Llonch-Masachs M., Heredero-Peris D., Montesinos-Miracle D., Rull-Duran J. \\ Centre d'Innovació Tecnològica en Convertidors Estàtics i Accionaments (CITCEA-UPC) \\ Departament d'Enginyeria Elèctrica, Universitat Politècnica de Catalunya \\ ETS d'Enginyeria Industrial de Barcelona, Av. Diagonal 647, 08028 Barcelona, Spain \\ Phone: +34 934016727 \\ Email: marc.llonch@ citcea.upc.edu, daniel.heredero@citcea.upc.edu, \\ montesinos@citcea.upc.edu
}

\section{Acknowledgments}

This work was supported by the Ministerio de Economía y Competitividad under the project ENE201347296-CE-2-R.

\section{Keywords}

$\ll$ Modulation Strategy $\gg, \ll$ Space Vector $\gg, \ll$ Three-phase system $\gg, \ll$ Voltage Source Converter $\gg$.

\begin{abstract}
This paper shows a new point of view of the classical voltage space vectors and its implications on three and four-leg converters. It is easy to find in the literature, authors using bi-dimensional and threedimensional representations of the converter states. Nonetheless, the literature rarely specifies what these spaces represent. Therefore, this paper proposes a wide analysis of the state voltages and its references for three-leg, three-leg four-wire and four-leg inverters, in favour of understanding the space vector behaviour under three and four-wire scenarios.
\end{abstract}

\section{Introduction}

Modulation techniques allow to provide the required gate signals for power electronics. For the control of power converters the most extended modulation technique is PWM (Pulse Width Modulation). The control of three-phase inverters under SVPWM (Space Vector-PWM) [1-3] has been proved to be more optimal than other modulation strategies as SPWM (Sinusoidal PWM). Its major advantages depend on the sequencing algorithm chosen and can be summarised into:

- Low output voltage harmonic distortion

- Good utilization of the available DC-link (up to $15.47 \%$ more than SPWM)

- Low switching losses

- Simple digital implementation in three-wire inverters

However, SVPWM advantages usability can depend on the inverter topology. In the particular case of three-phase inverters it is possible to consider:

- Three-wire inverters. Commonly used in case of renewable energy integration where the connection point is supposed to be balanced. This type of inverters are constituted by three-leg inverters (Fig. 1(a)).

- Four-wire inverters. Commonly used when the inverter needs to exchange energy with a low voltage grid, i.e. a four-wire grid or even a microgrid. This type of inverters can be classified into:

- Three-leg split capacitor topology (Fig. 1(b)).

- Four-leg topology (Fig. 1(c)). 


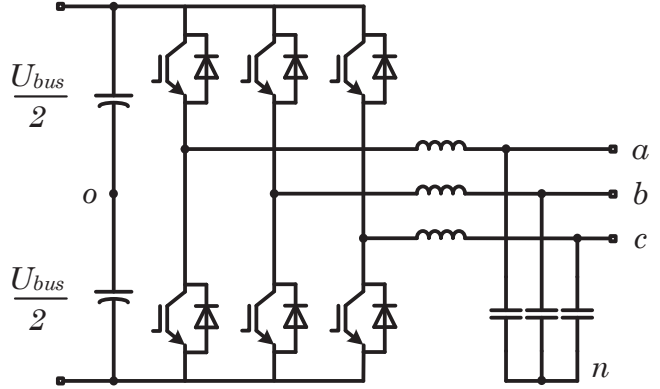

(a) Three-leg three-phase inverter

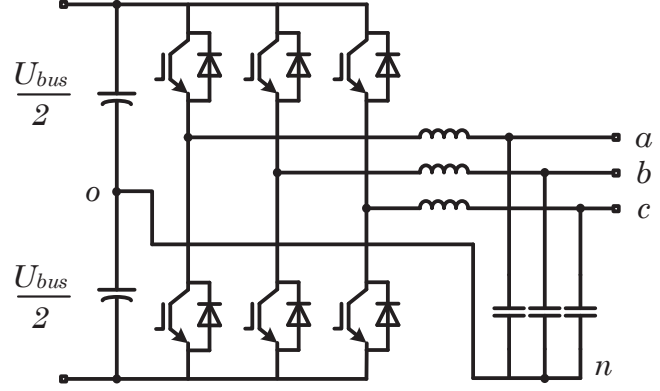

(b) Three-leg split capacitor

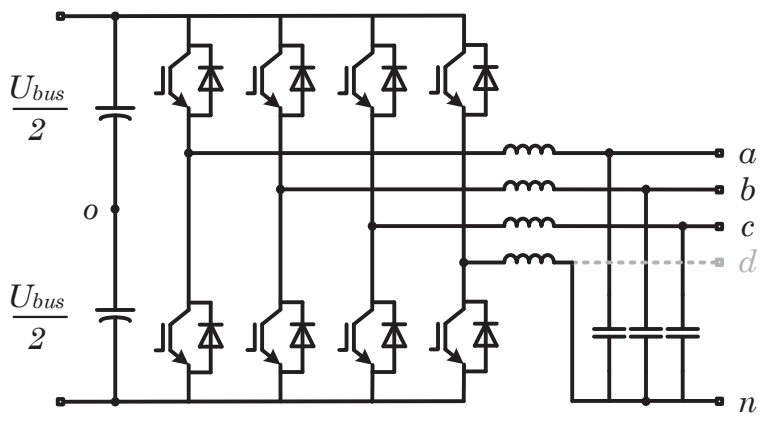

(c) Four-leg three-phase inverter

Fig. 1: Two-level three-phase inverter topologies

In this sense, the SVPWM has been widely studied in the literature for three and four-wire cases paying particular attention in different strategies to optimize the computational burden required to synthesize gate signals. This paper provides a conceptual analysis of how the SVPWM of a four-wire inverter can be understood from a three-wire scenario. To conduct this study it is important to remark that the reference concept is defined as the point from which the voltages are computed. Thus, when a reference frame is required [4] ( $a b c, \alpha \beta \gamma, d q 0)$, it will be only used frame to avoid confusions.

\section{SVPWM fundamentals}

\section{The space vector}

It is usual to depict three voltage phasors in positive sequence to represent a balanced three-phase system in steady-state. They can result ambiguous if it is desired to use these same phasors as axes to obtain a reference basis for an instantaneous vectorial analysis. This is due to the lineal dependency of the third component, i.e. there are only two degree of freedom.

Nevertheless, in a general unbalanced system where

$$
u_{a n}(t)+u_{b n}(t)+u_{c n}(t) \neq 0
$$

it can be deduced from (1) that, providing a vector moving in the space, it is possible to consider three degrees of freedom. The space vector $\mathbf{s}$ can be defined as a vector that represents each voltage at any

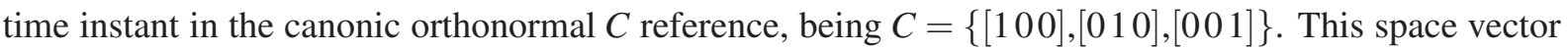
can be analysed from a stationary $a b c$ or $\alpha \beta \gamma$ frame, or from a synchronous $d q 0$ frame. These frames are obtained respectively, by the orthogonal Clarke's and Park's transform [4].

Then, it can be concluded that the space vector represents the instantaneous voltage values, in a basis which dimension matches with the number of degrees of freedom. 


\section{Three-leg inverter states}

In this case, a three-wire three-phase inverter is considered, as the one shown in Fig. 1(a). The SVPWM technique for a three-wire system, also known as 2D-SVPWM, is mainly based on the abstraction of considering the converter as a state machine where the desired output voltage is obtained from the superposition of the eight possible switching states. A switching vector is defined by a state represented by three digits (one per leg); if the digit is equal to one implies that the top switch of the corresponding leg is closed and if it is equal to zero that it is opened. The possible states and switching vectors are collected in Table I.

Table I: 2D-SVPWM vectors and states

\begin{tabular}{cccc}
\hline Vector & State & Vector & State \\
\hline V0 & 000 & V4 & 011 \\
V1 & 100 & V5 & 001 \\
V2 & 110 & V6 & 101 \\
V3 & 010 & V7 & 111 \\
\hline
\end{tabular}

These eight states are commonly drawn in the well known SVPWM hexagon of Fig. 2. This hexagon is divided into six sextants determined by V1 to V6. It should be noticed that exists two Zero Switching Vectors (ZSV) that corresponds to V0 and V7. These vectors produce null phase-to-phase voltages and are represented in the hexagon central point. The other six SV are also known as Non-ZSV (NZSV) [5].

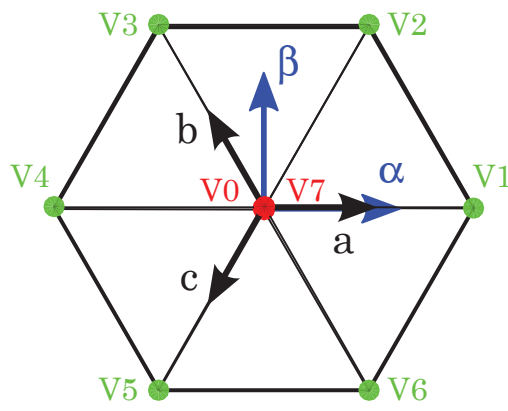

Fig. 2: Classic 2D representation of the three leg SVPWM.

Thus, SVPWM modulation can be computed as follows:

- Sextant determination. Selection of the two adjacent vectors $\mathbf{V}_{\text {adj }}$ according with the location of $\mathbf{s}$ in one of the sextants of the projected hexagon. Subscripts $x$ and $y$ represents the corresponding adjacent vectors of the hexagon at any time.

- Projection. Projection of $\mathbf{s}$ onto the selected $\mathbf{V}_{\mathrm{adj}_{x y}}$ in order to obtain $\operatorname{Proj}\left(V_{\mathrm{adj}_{x}} \mathbf{s}\right) \operatorname{and} \operatorname{Proj}\left(V_{\mathrm{adjj}_{y}} \mathbf{s}\right)$.

- Duty ratios calculation and selection of one sequencing for the switching vectors to obtain the gate signals.

\section{Four-leg inverter states}

In this case, a four-wire three-phase inverter is considered, as the one shown in Fig. 1(c). The SVPWM technique for a four-wire system, also known as 3D-SVPWM, is mainly based on the abstraction of considering the converter as a state machine where it is possible to obtain sixteen different vectors. In this case, each vector is represented by four digits; the three first ones are related with the active phases $\{a, b, c\}$ and the fourth with the neutral wire $\{n\}$. The possible states and switching vectors are collected in Table II. 
Table II: 3D-SVPWM vectors and states

\begin{tabular}{cccccccc}
\hline Vector & State & Vector & State & Vector & State & Vector & State \\
\hline V0 & 0000 & V4 & 0110 & V8 & 0001 & V12 & 0111 \\
V1 & 1000 & V5 & 0010 & V9 & 1001 & V13 & 0011 \\
V2 & 1100 & V6 & 1010 & V10 & 1101 & V14 & 1011 \\
V3 & 0100 & V7 & 1110 & V11 & 0101 & V15 & 1111 \\
\hline
\end{tabular}

These sixteen states are sometimes drawn conforming the dodecahedron of Fig. 3, which is integrated by the combination of two cubes connected by a common vertex constituted by V1 and V16. It should be noticed that exists two Zero Switching Vectors (ZSV) that corresponds to V0 and V15. The other fourteen SV are also known as Non-ZSV (NZSV).

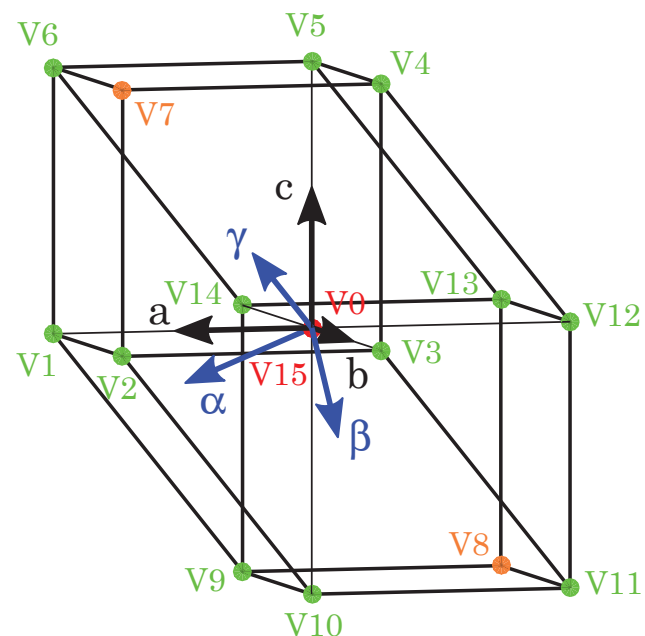

Fig. 3: 3D representation of the four leg SVPWM.

Thus, SVPWM modulation can be computed as follows:

- Tetrahedron determination. Selection of the three adjacent vectors $\mathbf{V}_{\text {adj }_{x y z}}$ according with the location of $\mathbf{s}$ in one of the twenty-four tetrahedrons. Subscripts $x, y$ and $z$ represents the corresponding adjacent vectors of the tetrahedron at any time.

- Projection. Projection of $\mathbf{s}$ onto the selected $\mathbf{V}_{\mathrm{adj}}$ ixz in order to obtain $\operatorname{Proj}\left(V_{\mathrm{adj} x} \mathbf{s}\right), \operatorname{Proj}\left(V_{\mathrm{adj} y} \mathbf{s}\right)$ and $\operatorname{Proj}\left(V_{\mathrm{adj}_{z}} \mathbf{s}\right)$.

- Duty ratios calculation and selection of one sequencing for the switching vectors to obtain the gate signals.

\section{Space vector of a three-leg inverter}

As has been aforementioned, in order to understand the dominion of a space vector, it is necessary to determine how many dimensions it has. In addition, this dominion also depends on the reference of the space vector voltages. Thus, two different scenarios can be extracted from the three-leg inverter: the space vector considering as a reference the midpoint of the DC-link $o$ or the AC neutral point $n$, depicted on Fig. 1(a).

It should be noticed that topics exposed immediately afterwards include the three-leg split capacitor topology. The voltages in this topology have only one possible reference: the midpoint of the DC-link $o$. Then, it generates the same space vectors as the three-leg three-phase inverter. 


\section{Space vector dominion on DC-link midpoint reference}

Considering the DC-link midpoint reference, it can be assumed that the space vector $\mathbf{s} \in \mathbb{R}^{3}$ due to its three degrees of freedom. Then, the inverter states of Table I conform the eight vertices of a cube in the space which edge is equal to the voltage applied at the DC-link side. Fig. 4 shows these eight states under the stationary $a b c$ frame.
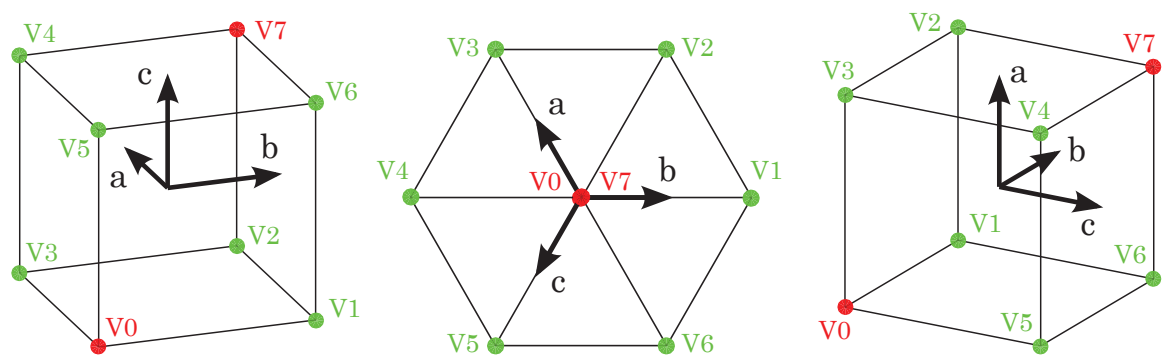

Fig. 4: Three-phase inverter voltage states referenced to the DC-link midpoint, in $a b c$ frame.

Notice that the cube boundaries are the physical limits of the maximum voltage space vector that could be generated by the inverter.

Drawing the space vector conformed by the maximum voltage balanced system, the Fig. 5(a) is obtained. Balanced system space vectors are always contained in a plane, named $\Pi$ in this paper, which normal vector is $\vec{n}=(1,1,1)$. The special characteristic of $\Pi$ is that all vectors contained do not have Zero Sequence component. Fig. 5(b) shows the null ZS part by applying the Clarke's transformation, changing the $a b c$ frame to the $\alpha \beta \gamma$ frame.
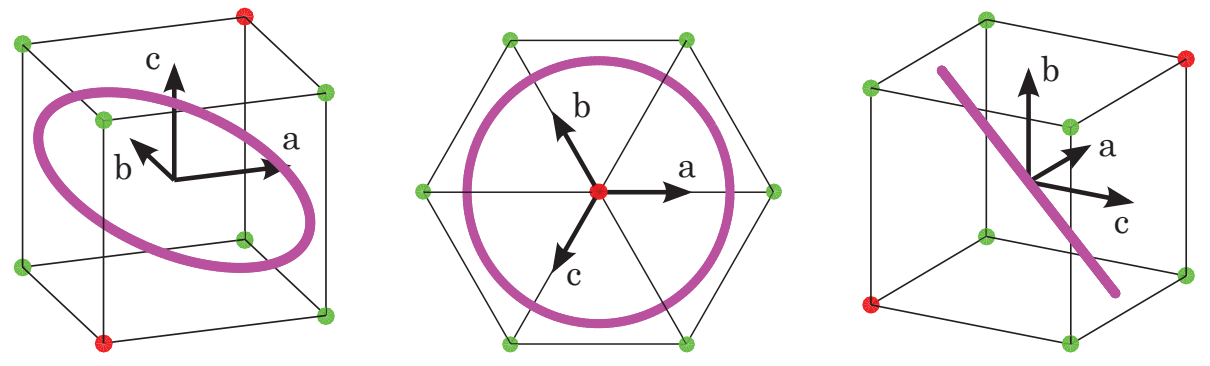

(a) In $a b c$ frame.
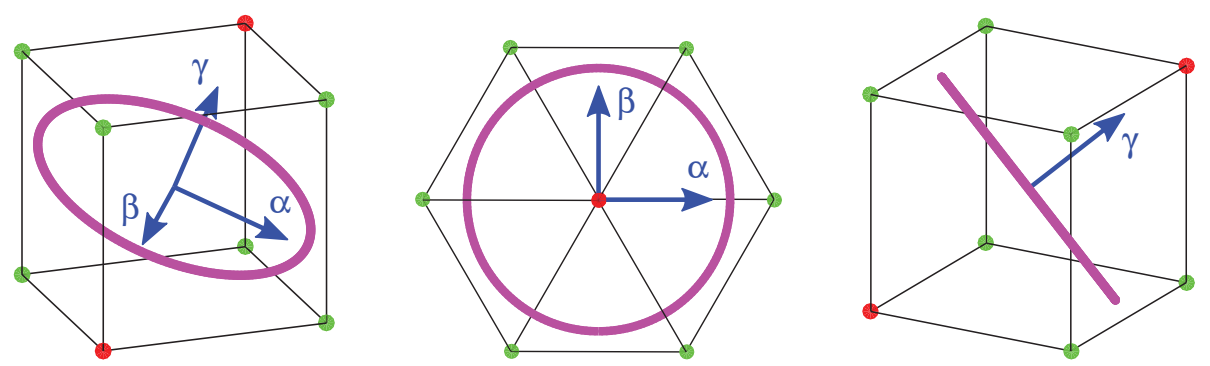

(b) In $\alpha \beta \gamma$ frame.

Fig. 5: Three-phase balanced space vector referenced to the DC-link midpoint.

\section{Space vector dominion on AC neutral point reference}

Considering the neutral point $n$ as a reference, one degree of freedom is lost due to $n$ is floating in respect with $o$. It is widely known that Zero Sequence components cannot be generated from a floating neutral point reference. Fig. 6 shows how the inverter voltage states are moved, losing the homopolar dimension and forcing $\mathbf{s} \in \mathbb{R}^{2}$. This fact can be explained as follows. 

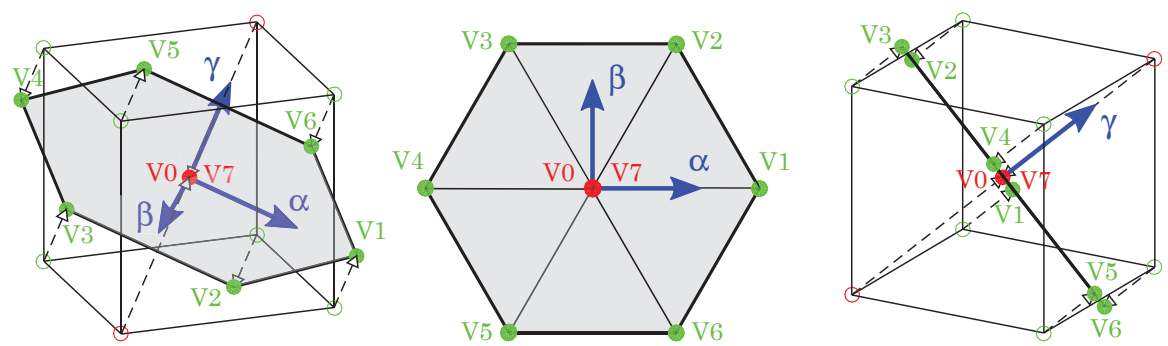

Fig. 6: Three-phase inverter voltage states referenced to the AC neutral point, in $\alpha \beta \gamma$ frame.

Assuming the converter states vectors referenced to $o$ as

$$
\vec{E}_{i}^{o}=\left(u_{a o}, u_{b o}, u_{c o}\right)
$$

these can be referenced to $n$ by

$$
\vec{E}_{i}^{n}=\vec{E}_{i}^{o}-\vec{u}_{n o}
$$

where

$$
\vec{u}_{n o}=\frac{u_{a o}+u_{b o}+u_{c o}}{3} \cdot(1,1,1)
$$

In order to give more information, SVPWM notation has been changed from $V 0, V 1, V 2 \ldots$ to $\vec{E}_{i}^{r}$ where $i$ is the vector number and $r$ represents the system reference: $o$ for the DC-link midpoint and $n$ for the AC neutral point.

\section{Space vector under different modulations and references}

As it has been introduced, SPWM and SVPWM strategies are widely used modulations to control threephase inverters. Taking into account the space vector dominions mentioned above, the space vector trajectory plots can significantly increase the strengths and weaknesses comprehension of these strategies.

Fig. 5(a) shows the average space vector trajectory using SPWM, referenced to the $o$ point. It can be seen how the intersection of $\Pi$ plane with the cube determines the maximum voltage avoiding overmodulation. In that case, the $\vec{n}$ view (central plot of Fig. 5(a)) also can represent the same trajectory, referenced to the neutral point $n$.

Using SVPWM, the three-dimensional point of view results useful to understand why it is possible to overtake in a $15.47 \%$ the maximum voltage. As it can be seen in Fig. 7 , the space vector trajectory referenced to the neutral point $n$ is the expected balanced system circle. However, referencing to the DC-link midpoint $o$, it can be seen how takes more profit of the DC-link by using the Zero Sequence component.
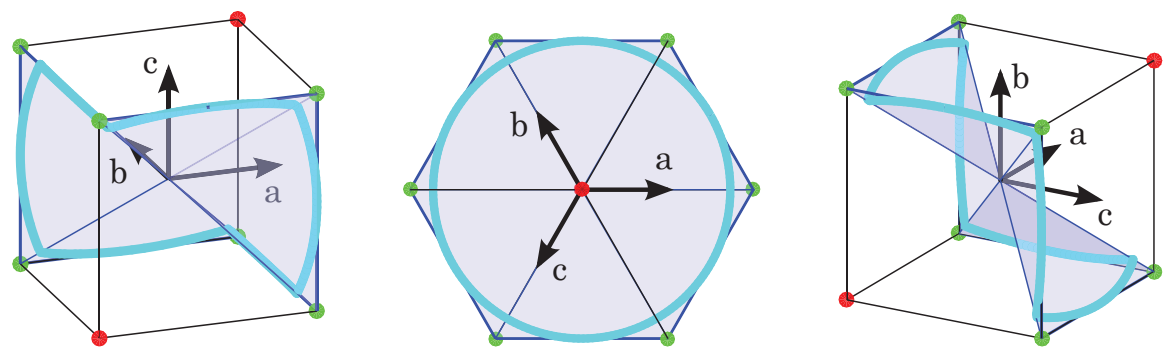

Fig. 7: Maximum space vector trajectory referenced to the DC-link midpoint, by using SVPWM. 


\section{Space vector dominion after a phase-neutral short-circuit}

The three-phase inverter space vector dominion after a single-phase short-circuit is practical to understand the four-phase inverter.

After a short-circuit between the $c$ phase and the neutral point $n$, the converter states referenced to $o$ of (2) become referenced to $n$ as

$$
\vec{E}_{i}^{n}=\vec{E}_{i}^{o}-\vec{u}_{c o}
$$

where

$$
\vec{u}_{c o}=u_{c o} \cdot(1,1,1)
$$

Then, the space vector dominion shown in Fig. 8 is obtained. The converter states suffer a displacement through the $\vec{n}$ direction, to a plane $\Pi_{2} \mid\{c=0\}$. In other words, a bi-dimensional space appears as a conjunction of two bi-phase dominions (squares). One represents when the inverter connects the shortcircuited phase to the negative DC-link point and the other when it is connected to the positive one.

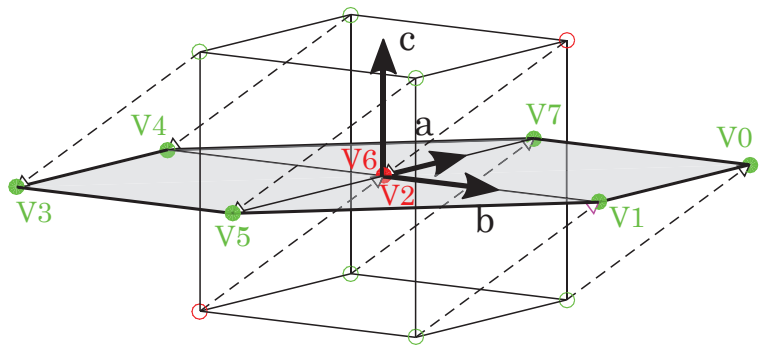

Fig. 8: Three-phase inverter voltage states after a mono-phase short-circuit.

\section{Space vector of a four-leg inverter}

Analysing the four-leg inverter showed in Fig. 1(c) in the same way as the three-leg one, two scenarios can also be extracted: the space vector considering as a reference the midpoint of the DC-link $o$ and the space vector considering as a reference the AC neutral point $n$.

\section{Space vector dominion on DC-link midpoint reference}

Considering the DC-link midpoint reference, it can be assumed that the space vector $\mathbf{s} \in \mathbb{R}^{4}$ due to its four degrees of freedom. Then the inverter states of Table II conform the sixteen vertices of a hipercube or tesseract in the tetra-dimensional space which edge is equal to the voltage applied at the DC-link side. Fig. 9 shows a projection of these sixteen states under the stationary abcd frame.

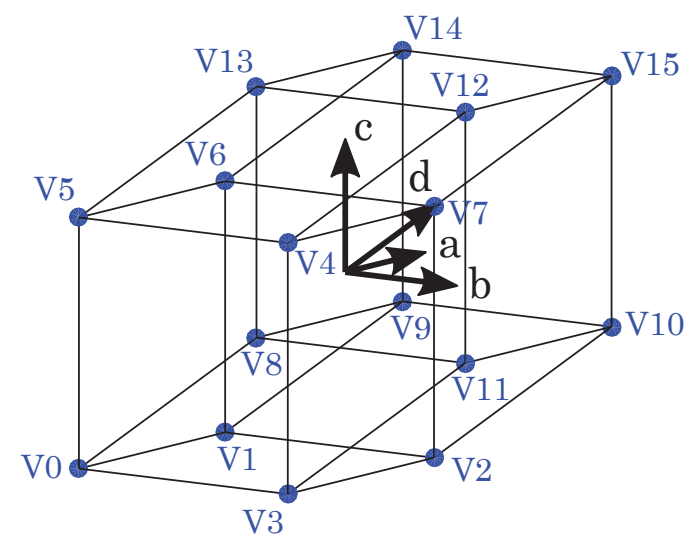

Fig. 9: Four-phase inverter voltage states referenced to the DC-link midpoint, in abcd frame. 


\section{Space vector dominion on AC neutral point reference}

Considering the neutral point $n$ as a reference, one degree of freedom is lost. It this case, the reason is a deliberated short-circuit between the $d$ phase and the AC neutral point $n$. At this point, the analysis of the phase to neutral short-circuit aforementioned becomes helpful.

Making an analogy of what happens in the three-dimensional dominion to the four dimensional case, the four-leg converter states can be drawn in a three-dimensional space.

Assuming the converter states vectors referenced to $o$ as

$$
\vec{E}_{i}^{o}=\left(u_{a o}, u_{b o}, u_{c o}, u_{d o}\right)
$$

these can be referenced to $n$ as

$$
\vec{E}_{i}^{n}=\vec{E}_{i}^{o}-\vec{u}_{d o}
$$

where

$$
\vec{u}_{d o}=u_{d o} \cdot(1,1,1,1)
$$

Then, the fourth dimension is cancelled and the space vector dominion of Fig. 10(a) is obtained. Converter states suffer a displacement through the four-dimension homopolar vector $\vec{m}=(1,1,1,1)$ to a volume $\Omega \mid\{d=0\}$. In other words, a three-dimensional space appears as a conjunction of two threephase dominions (cubes). One represents when the inverter connects the short-circuited phase to the negative DC-link point, and the other when it is connected to the positive DC-link point.

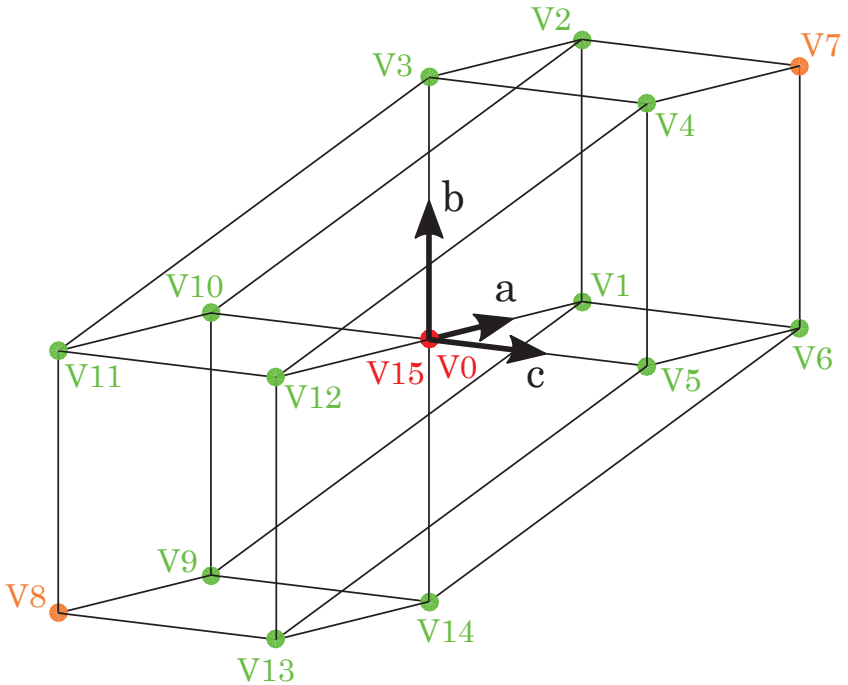

(a) $3 \mathrm{D}$ view.

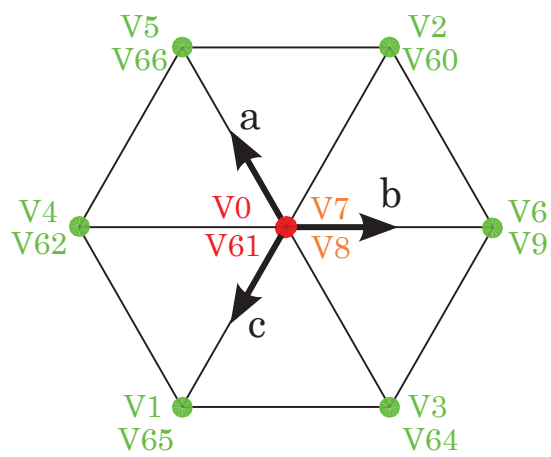

(b) $(1,1,1)$ view.

Fig. 10: Four-phase inverter voltage states referenced to the AC neutral point.

As it can be seen in Fig. 10(a), also in Fig. 3, there exists two ZSV (V0 and V15) which have a null space vector referenced to $n$. More over, there also exists two NZSV (V7 and V8) which are only able to generate homopolar component. Finally, there exists twelve NZSV capable to generate space vectors projections in the $\Pi$ plane. As it can be seen in Fig. 10(b), in order to generate balanced systems, the four-leg inverter is similar to the three-leg one. Nevertheless, four-leg have two SV for each $\Pi$ state, one with positive homopolar and the other in negative. Thus, four-leg inverters waveforms will have less high frequency switching harmonics, apart from a higher capability to produce homopolar content. 


\section{Controllability region}

Once analysed the space vector dominion, it is important to highlight that in terms of controllability, it is not $100 \%$ usable [6]. The converter output voltages have two components: one large component that matches the output voltage and a second incremental one used to manage the output current. Then, in order not to have controllability changes throughout the voltage phase, the space vector dominion is constrained to a symmetrical region in respect with the $\vec{n}$ direction.

Fig. 11(a) shows the controllability limit in blue, for a three-leg three-phase inverter (Fig. 1(a)). It is a circle that enclose the bi-dimensional space vector dominion referenced to the $\mathrm{AC}$ neutral point.

Fig. 11(b) shows the controllability limit in blue, for a three-leg split capacitor inverter (Fig. 1(b)). It is the union of two cones that enclose the three-dimensional space vector dominion referenced to the $\mathrm{AC}$ neutral point.

Fig. 11(c) shows the controllability limit in blue, for a four-leg inverter (Fig. 1(c)). It is a cylinder closed by two cones, that enclose the three-dimensional space vector dominion referenced to the AC neutral point.

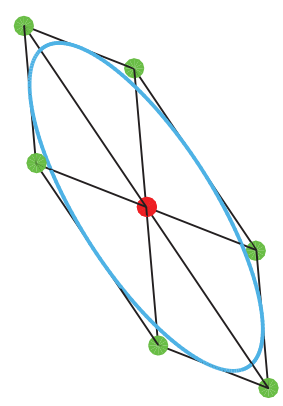

(a) Three-leg threephase inverter.

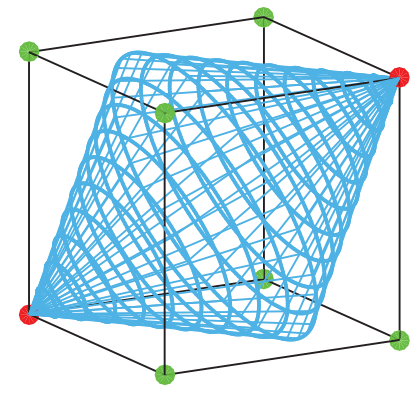

(b) Three-leg split capacitor inverter.

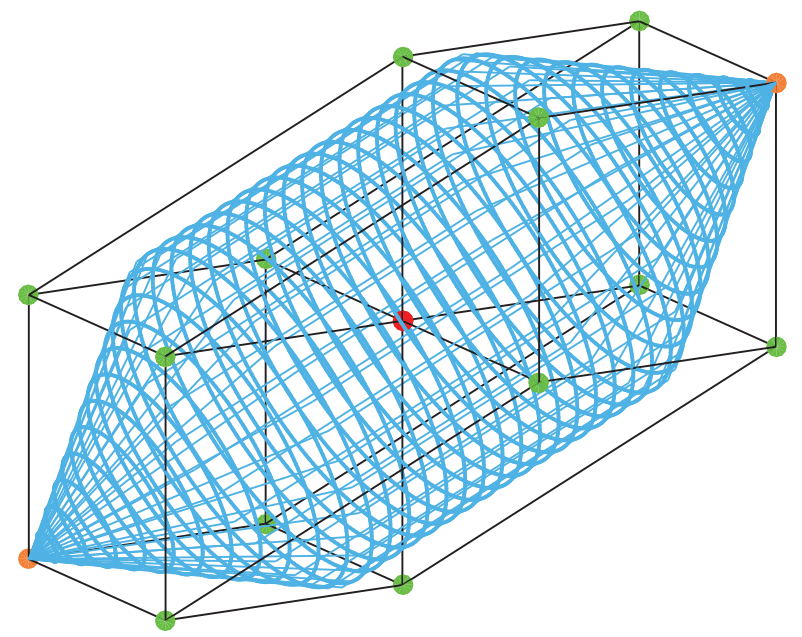

(c) Four-leg three-phase inverter.

Fig. 11: Space vector controllability limits depending on the topology.

Overlaying the controllability regions on Fig. 12, some differences of the principal three-phase inverter topologies (Fig. 1) can be highlighted.

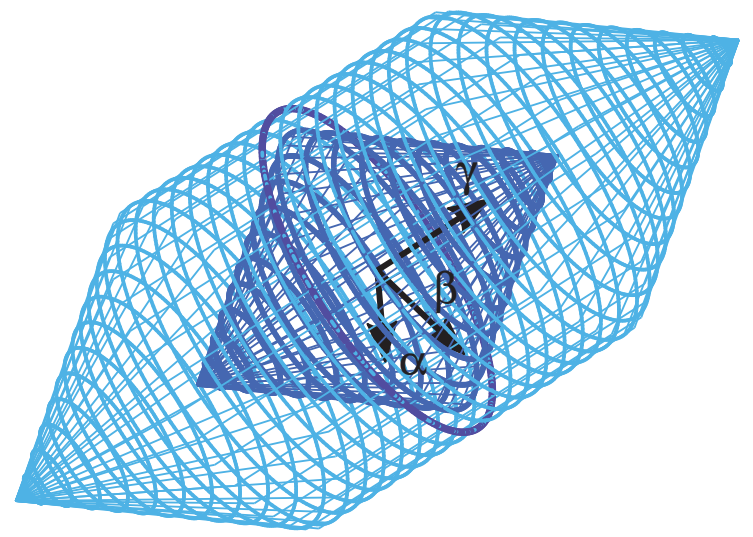

Fig. 12: Overlaying the three-phase space vector controllability limits. 
- The three-leg three-phase inverter can have a good utilization of the available DC-link due to the voltage vector projections on the $\alpha \beta$ plane. Therefore, it can generate larger output voltages (up to $15.47 \%$ more, if using SVPWM), not being able to obtain homopolar component.

- The three-leg split capacitor inverter cannot use SVPWM, then it can generate lower voltages than the previous one. The fourth wire makes possible the homopolar current, that can unbalance the DC-link.

- The four-leg three-phase inverter has the major controllability region. It can synthesise balanced voltages, in the $\alpha \beta$ plane, as larger as the three-leg three-phase inverter. Furthermore, it can also generate unequal voltages with larger homopolar component than the three-leg split capacitor, without unbalancing the DC-link.

\section{Conclusions}

This paper has exposed the influences of the voltage reference and the three and four-leg inverter topologies on the space vector dominion. It has given a wider point of view of the space vector trajectories through its $n$-dimensional spaces, useful to understand the strengths and weaknesses of different modulation strategies. It has been shown the inverter voltage states referenced to the DC-link and to the AC neutral point. Then it has also been presented the differences between SPWM and SVPWM on its space vector trajectory.

Finally, the controllability limits has been plotted and compared to give a graphical perception of different inverter topology capabilities.

\section{References}

[1] H. Akagi, Y. Tsukamoto, and A. Nabae, "Analysis and design of an active power filter using quadseries voltage source PWM converters," Industry Applications, IEEE Transactions on, vol. 26, no. 1, pp. 93-98, 1990.

[2] J. W. Kolar, E. R. T. L. Hans, and C. Zach, "Influence of the modulation method on the conduction and switching losses of a pwm converter system," vol. 21, no. 6, pp. 1063-1075, 1991.

[3] P. Enjeti and B. Xie, "A new real time space vector PWM strategy for high performance converters," Conference Record of the 1992 IEEE Industry Applications Society Annual Meeting, no. i, pp. 10181024, 1992.

[4] G. C. Paap, "Symmetrical Components in the Domain and Their Application to Power Network Calculations," IEEE Transactions on power systems, vol. 15, no. 2, 2000.

[5] R. Zhang, V. H. Prasad, D. Boroyevich, and F. C. Lee, "Three-dimensional space vector modulation for four-leg voltage-source converters," IEEE Transactions on Power Electronics, vol. 17, no. 3, pp. 314-326, 2002.

[6] D. Shen and P. Lehn, "Fixed-frequency space-vector-modulation control for three-phase four-leg active power filters," IEE Proceedings - Electric Power Applications, vol. 149, p. 268, 2002. 